\title{
Evaluación de la motricidad y el carácter de los alumnos de 4 y 5 años: un estudio piloto
}

\section{Evaluation of motor skills and character of children aged 4 and 5 years old: a pilot study}

\author{
Rosa María López Esparcia, Alejandro Prieto-Ayuso, María Pilar León González* y Pedro Gil-Madrona
}

Universidad de Castilla-La Mancha (España).

\begin{abstract}
Resumen: El objetivo fue evaluar la motricidad gruesa y el carácter en niños de 4 y 5 ańos. Para ello, se seleccionaron 23 participantes y se aplicaron la subprueba locomotriz y la de control de objetos, ambas pertenecientes al Test of Gross Motor Development (TGMD 2), así como una rúbrica sobre carácter (respeto, cooperación, sensibilidad, liderazgo, trabajo en equipo y autocontrol). Los resultados muestran que las chicas son mejores en la subprueba locomotriz, mientras que los chicos destacan en el control de objetos. En general, los niños tienen menos habilidad en el salto extendido y en la tarea de atrapar objetos. En cuanto al carácter, los chicos son peores en el liderazgo y las chicas en el trabajo en equipo. Por tanto, se concluye la necesidad de hacer hincapié específicamente en aquellos aspectos donde menor puntuación obtuvieron. Como prospectiva de investigación, este trabajo debe ser realizado con muestras más grandes para conocer si los resultados se mantienen similares.
\end{abstract}

Palabras clave. Desarrollo motor, carácter, niños, educación, infancia. Abstract: The aim was to assess gross motor and character in children aged 4 and 5 years old. For this, 23 participants were selected and the locomotive and object control sub-tests were applied, both belonging to the Test of Gross Motor Development (TGMD 2), as well as a rubric of character (respect, cooperation, sensitiveness, leadership, teamwork, and self-control) Results show that girls are better in the locomotive subtest, while boys excel in the object control. In general, boys have less skill in the extended jumping and in the objects catch task. In terms of character, boys are worse in leadership and girls in teamwork. Therefore, it is important to emphasize especially those motor skills and emotional aspects where children score lower. As research prospect, this study must be conducted with a bigger sample to know if these results remain similar.

Key words. Motor development, character, children, education, childhood

\section{Introducción}

El desarrollo del niño es un proceso evolutivo multidimensional mediante el cual va adquiriendo la autonomía e independencia propia del adulto. Durante los primeros ańos de vida este desarrollo es muy rápido e importante, ya que sienta las bases de futuros aprendizajes, y está estrechamente relacionado con la cantidad y calidad de las estimulaciones a las que se ven sometidos los aprendices (Gabbard, Caçola, Spesatto, \& Santos, 2012). En este desarrollo, por tanto, juega un papel crucial la Educación Infantil, debido a que es el primer periodo de educación institucionalizada, donde la motricidad comienza su desarrollo (Bornstein, Hahn, \& Suwalsky, 2013; Inverson, 2010). Por tanto, es fundamental que desde el ámbito educativo se ofrezca a los niños diferentes medios que les ayuden a establecer distintas habilidades motrices básicas que seguirán siendo desarrolladas a lo largo de la infancia (True et al., 2017). Así, los nińos podrán lograr una competencia motriz adecuada, la cual también es fundamental para el desarrollo cognitivo y social de éstos (Morrison, 2005).

La principal finalidad de este periodo educativo es contribuir al desarrollo físico, afectivo e intelectual del niño,

\footnotetext{
Dirección para correspondencia [Correspondence address]: María Pilar León González. Universidad de Castilla-La Mancha (España).

E-mail: MariaPilar.Leon@uclm.es
}

dándole especial relevancia a los aprendizajes orientados al conocimiento, valoración y control del cuerpo, de sus posibilidades y de la capacidad para utilizar con cierta autonomía e independencia los recursos disponibles en cada momento. El trabajo globalizado a partir del ámbito motor puede optimizar el desarrollo del esquema corporal, los aspectos perceptivo-motrices, la higiene postural, la expresión de emociones, la creatividad y las habilidades sociales (SanzArazuri, Alonso-Ruíz, Valdemoros, \& Ponce de León, 2013), lo que es amparado en la Ley Orgánica para la Mejora de la Calidad Educativa (LOMCE, 2013). En ella, se afirma que la etapa de Educación Infantil cumple con una función preparatoria, debido a que da acceso a la enseñanza obligatoria y compensatoria, y permite abordar los problemas precozmente, lo cual resulta decisivo para la vida del niño. Asimismo, el artículo 2 de la Orden de 12/05/2009, señala que la evaluación es una herramienta clave, cuya finalidad consiste en identificar los aprendizajes adquiridos, determinar el grado de consecución de los objetivos previstos y conocer el nivel de desarrollo alcanzado en las competencias básicas, así como el ritmo y características de la evolución de cada niño, tomando como referencia los criterios de evaluación de cada una de las áreas.

Gil-Madrona (2004) advierte que la evaluación en Edu- 
cación Física infantil puede servir como elemento preventivo, con la finalidad de logar un conocimiento lo más exhaustivo posible de la evolución del alumno y, de este modo, adecuar el proceso educativo al niño. Otra de las cualidades de esta evaluación es la mejora del aprendizaje a través de actividades que determinen el nivel de madurez motriz del niño, para lo cual es necesario que el docente conozca el nivel de desarrollo motor de sus alumnos en cada momento.

Con la valoración de dicha motricidad, el docente también podrá conocer la evolución de éstos, teniendo en cuenta cuatro aspectos: la identificación, es decir, el reconocimiento del progreso en el aprendizaje del niño; el diagnóstico, con el propósito de analizar la situación en la que se realizan las tareas motrices; la orientación, para saber cómo ayudar al alumno a mejorar y solucionar problemas motrices que se planteen; y por último, la información, con el fin de obtener datos objetivos acerca de la evolución de la motricidad.

El desarrollo motor tiene una gran influencia en el crecimiento general del niño durante sus primeros años de vida, produciéndose con el tiempo cambios en la conducta motora, que reflejan la interacción del organismo humano con el medio y que parte de las conductas motrices humanas innatas (Batalla, 2000). Con la maduración, el nińo adquiere patrones de comportamiento que le ayudan a desarrollarse y a adquirir gradualmente la maduración necesaria para alcanzar una motricidad más específica. En este sentido, Coleto (2009) indica que el desarrollo de las habilidades motrices depende de la maduración neurológica y pasa por las siguientes fases:

- Fase de automatismo: corresponde con los primeros meses donde la mayoría de las acciones son reflejas.

- Fase receptiva: se extiende a lo largo del segundo trimestre de vida y coincide con el perfeccionamiento de los sentidos. Las acciones ya son voluntarias, aunque predomina la observación del entorno a través de los cinco sentidos.

- Fase de experimentación y adquisición de conocimientos: comienza en los primeros meses y se extiende a lo largo de toda la vida. Las habilidades motrices se utilizan como medio para adquirir conocimiento.

Dentro del ámbito escolar, y debido a la importancia que tiene la motricidad en los niños, recientes estudios han tratado de abordar cómo se produce este desarrollo motor en alumnos de Educación Infantil y Primaria (Cuesta, Prieto-Ayuso, \& Gil Madrona, 2016; Madrid, Prieto-Ayuso, Samalot-Rivera, \& Gil-Madrona, 2016; Saiz-Panadero, Prieto-Ayuso, Gutiérrez, \& Gil-Madrona, 2016), así como la evolución producida en las habilidades sociales y emociones, debido a su gran importancia para el niño. Según Denham, Susanne, Bassett, Hideko y Zinsser (2012), aquellos que no tienen una adecuada regulación emocional enfrentan ciertos problemas en las áreas del aprendizaje, ya que dicha regulación está conectada con una afectividad social positiva por parte de los niños. Por esta razón, la evaluación del carácter se precisa fundamental desde las primeras edades, con el fin de evitar problemas futuros, tanto en su desarrollo intra como interpersonal.

Así, por ejemplo, en el estudio llevado a cabo por Cuesta et al. (2016), se plantearon como objetivo evaluar las habilidades motrices y sociales a través de un diseño transversal, y propusieron también el trabajo conjunto de ambos aspectos. Los resultados mostraron que los elementos afectivo-relacionales están muy poco desarrollados y es donde los docentes deberían focalizar la atención educativa. Por su parte, Madrid et al. (2016) realizaron una medición antes y después de haber puesto en práctica un programa para la mejora de las conductas apropiadas en la enseńanza de los deportes, concluyendo la necesidad de más programas de intervención validados que ayuden a la mejora en la adquisición de habilidades sociales por parte del alumnado. En último lugar, cabe destacar el trabajo de Saiz-Panadero et al. (2016) en el que evaluaron las habilidades sociales en alumnos de Educación Primaria, a través del Cuestionario de Habilidades de Interacción Social (CHIS). Como resultados, obtuvieron que la habilidad menos desarrollada era la de hacer amigos, mientras que las habilidades básicas de interacción social fueron las que obtuvieron mejores puntuaciones. En cuanto al género, las chicas mostraron mejor puntuación media que los chicos. Como conclusión principal del estudio se destaca la necesidad de trabajar las habilidades sociales dentro de esta etapa educativa, puesto que resultan fundamentales para desenvolverse en la sociedad.

Por tanto, y en virtud de lo expuesto hasta el momento, parece obvio pensar que las relaciones afectivas establecidas en situaciones de actividad motriz, y en particular mediante el juego, serán fundamentales para el crecimiento emocional (Gil-Madrona, 2003). Por esta razón, el objetivo del trabajo no ha sido otro que el de conocer el estado de la motricidad gruesa y el carácter de un grupo de niños de 4 y 5 años.

\section{Método}

\section{Participantes}

La muestra se seleccionó de forma no aleatoria por muestreo incidental, debido a la facilidad de acceso al centro educativo. El colegio estaba ubicado en una localidad rural de la provincia de Albacete. La muestra estuvo compuesta por 23 estudiantes, divididos en 12 niños (52.2\%) y 11 nińas (47.8\%) de entre 4 y 5 ańos. 


\section{Instrumentos}

Para la recogida de datos se utilizaron los siguientes instrumentos procedentes de la New York State Association for Health, Physical Education, Recreation and Dance (2015):

Test of Gross Motor Development (TGMD 2). Este test fue administrado con el propósito de medir el desarrollo motor. Según Ulrich (2000) la prueba puede ser utilizada fundamentalmente en:

- Identificar a los niños que tienen un retraso considerable en el desarrollo de las habilidades motrices.

- Planificar un programa de instrucción en el desarrollo de las habilidades motrices.

- Evaluar los progresos individuales en el desarrollo motor.

- Evaluar el éxito del programa de motricidad gruesa.

- Servir como un instrumento de medición en investigaciones sobre el desarrollo motor grueso.

La prueba se compone de dos subpruebas que pretenden medir la coordinación motora de los niños, a través de doce destrezas de movimiento fundamentales:

- Subprueba locomotriz. Tiene por objeto medir las habilidades motoras gruesas que requieren de movimientos coordinados del cuerpo del niño (Ulrich, 2000). Éstas son:

o Correr

o Galopar

o Brincar con un pie

o Salto extendido

o Salto horizontal

o Deslizamiento

- Subprueba control de objetos. Se pretende medir la capacidad general de los niños en las habilidades manipulativas. Las seis competencias que conforman la subprueba del aparato locomotor son:

o Golpeo (lanzamiento con el brazo bajo y lanzamiento con el brazo)

o Driblar

o Atrapar

o Patear

o Tiro (golpe corto y largo)

o Rodada (voleo de brazo bajo y voleo de cabeza)

La puntuación obtenida de estas pruebas fue cuantitativa, puntuando un 0 si lo realizaba incorrectamente y un 1 si lo realizaba correctamente.
K-2 Rúbrica sobre carácter. La rúbrica de carácter K-2 valora el dominio afectivo a través de seis categorías, para cada una de las cuales se selecciona, de entre 5 opciones, el nivel en el que se encuentra el nińo, siendo el de mayor frecuencia "consistentemente" y el de menor frecuencia "nunca". El consentimiento para la utilización de este instrumento ha sido concedido por la Asociación Americana para la Salud, Educación Física, Recreación y Danza (AAHPERD), contando con la traducción de manos de un profesor de Kinesiología de la universidad de Nueva York. Las seis categorías son las siguientes:

o Respeto. Reconocer el rol y seguir las reglas en las actividades físicas diseñadas. Comprender y aceptar el rol de cada participante y cumplir las normas establecidas para el desarrollo de la actividad física diseñada.

o Cooperación. Trabajar de manera colaborativa con otros compañeros en distintos entornos. Colaborar con otros compañeros para lograr, a través de una tarea, el resultado deseado. Para ello, es necesaria la implicación individual de cada miembro, reconociendo el rol de cada uno y su importancia dentro del grupo de trabajo.

o Sensibilidad. Trabajar con compańeros que son diferentes al resto. Comprender y respetar las diferencias individuales, mostrando predisposición para trabajar con cualquier compañero.

o Liderazgo. Participar de manera constante en actividades desafiantes con esfuerzo para lograr el éxito. Participar activamente en las tareas propuestas, siendo capaz de gestionar el propio grupo y dirigir las tareas de todos los miembros hacia el logro del objetivo.

o Trabajo en equipo. Cooperación con el resto de compańeros para conseguir una tarea. Desarrollar diferentes tareas con la ayuda de los compañeros que componen el grupo de trabajo.

o Autocontrol. Acepta su responsabilidad y sabe comportarse en cualquiera de las situaciones acaecidas durante cualquier actividad. Ser capaz de gestionar sus emociones y comportamiento ante cualquier situación ocurrida en el desarrollo de la actividad, adoptando una actitud adecuada y llevando a cabo la responsabilidad asignada.

La puntuación obtenida de esta prueba fue cuantitativa, a través de una escala Likert de 4 puntos, siguiendo la leyenda mostrada en la Tabla 1. 
Tabla 1. Escala Likert del instrumento K-2 Rúbrica sobre el carácter.

Respeto

1 Ocasionalmente reconoce su rol en seguir las reglas y la etiqueta (conducta) en las actividades físicas diseñadas por el maestro. Ocasionalmente muestra respeto por el maestro y sus compañeros siguiendo las instrucciones, reglas y rutinas de clase, así como respetando el equipo utilizándolo.

2 Inconsistentemente reconoce su rol en seguir las reglas y la etiqueta (conducta) en las actividades físicas diseñadas por el maestro. Inconsistentemente muestra respeto por el maestro y sus compańeros siguiendo las instrucciones, reglas y rutinas de clase, así como respetando el equipo utilizándolo apropiadamente.

3 Frecuentemente reconoce su rol en seguir las reglas y la etiqueta (conducta) en las actividades físicas diseñadas por el maestro. Frecuentemente muestra respeto por el maestro y sus compañeros siguiendo las instrucciones, reglas y rutinas de clase, así como respetando el equipo utilizándolo apropiadamente.

4 Consistentemente reconoce su rol en seguir las reglas y la etiqueta (conducta) en las actividades físicas diseñadas por el maestro. Constantemente muestra respeto por el maestro y sus compañeros siguiendo las instrucciones, reglas y rutinas de clase, así como respetando el equipo utilizándolo apropiadamente.

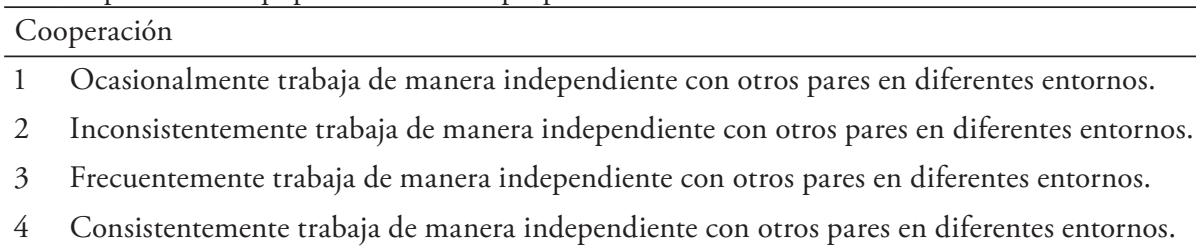

Sensibilidad

1 Ocasionalmente trabaja (juega) con un compańero que de alguna manera es diferente a ellos mismos.

2 Inconsistentemente trabaja (juega) con un compañero que de alguna manera es diferente a ellos mismos.

3 Frecuentemente trabaja (juega) con un compañero que de alguna manera es diferente a ellos mismos.

4 Consistentemente trabaja (juega) con un compañero que de alguna manera es diferente a ellos mismos.

\begin{tabular}{|c|c|}
\hline \multicolumn{2}{|c|}{ Liderazgo } \\
\hline 1 & Ocasionalmente persiste participar en actividades que son desafiantes y se esfuerza para lograr el éxito. \\
\hline 2 & Inconsistentemente persiste participar en actividades que son desafiantes y se esfuerza para lograr el éxito. \\
\hline 3 & Frecuentemente persiste participar en actividades que son desafiantes y se esfuerza para lograr el éxito. \\
\hline 4 & Consistentemente persiste participar en actividades que son desafiantes y se esfuerza para lograr el éxito. \\
\hline \multicolumn{2}{|c|}{ Trabajo en equipo } \\
\hline & Ocasionalmente coopera con un compañero para completar una tarea. \\
\hline & Inconsistentemente coopera con un compañero para completar una tarea. \\
\hline & Frecuentemente coopera con un compañero para completar una tarea. \\
\hline & Consistentemente coopera con un compańero para completar una tarea. \\
\hline \multicolumn{2}{|c|}{ Auto-control } \\
\hline & $\begin{array}{l}\text { Ocasionalmente practica destrezas con un mínimo de recordatorios del maestro. } \\
\text { Inconsistentemente acepta la responsabilidad de los protocolos de clase con acciones de comportamiento y rendimiento adecuados. }\end{array}$ \\
\hline & $\begin{array}{l}\text { Inconsistentemente practica destrezas con un mínimo de recordatorios del maestro. } \\
\text { Inconsistentemente acepta la responsabilidad de los protocolos de clase con acciones de comportamiento y rendimiento adecuados. }\end{array}$ \\
\hline & $\begin{array}{l}\text { Frecuentemente practica destrezas con un mínimo de recordatorios del maestro. } \\
\text { Frecuentemente acepta la responsabilidad de los protocolos de clase con acciones de comportamiento y rendimiento adecuados. }\end{array}$ \\
\hline & $\begin{array}{l}\text { Consistentemente practica destrezas con un mínimo de recordatorios del maestro. } \\
\text { Consistentemente acepta la responsabilidad de los protocolos de clase con acciones de comportamiento y rendimiento adecuados. }\end{array}$ \\
\hline
\end{tabular}

\section{Procedimiento}

Los datos fueron tomados en horario lectivo durante nueve días, siendo los encargados de ello dos investigadores y la tutora del aula. Una vez pasado el TGMD 2, se procedió a la elaboración y recogida de datos de las rúbricas correspondientes al carácter, cumplimentada por la maestratutora. 
Para las pruebas locomotrices del TGMD 2 se utilizaron conos con el fin de controlar la distancia entre los niños durante la realización de dichas pruebas. Por otro lado, para las pruebas referentes al control de objetos se utilizó una bola plástica estacionaria de 4 pulgadas, bate de plástico y base de bateo, bola de 8-10 pulgadas o de baloncesto, bola de futbol, tenis y conos.

\section{Análisis estadístico}

El análisis de los resultados se realizó mediante el programa estadístico SPSS v. 22.0. Se calcularon las frecuencias y las medias ponderadas, con el propósito de conocer cuál era el estado de motricidad gruesa y el carácter de un grupo de alumnos de Educación Infantil.

\section{Resultados}

Con el propósito de dar respuesta al objetivo de investigación marcado para este trabajo, en primer lugar, se hallaron los descriptivos básicos sobre el carácter que poseían este grupo de niños de Educación Infantil. Así, en la Figura 1, se comprueba que la sensibilidad (3.78) es el aspecto que mayor puntuación recibió, mientras que la peor puntuación fue marcada por el liderazgo (3.08).

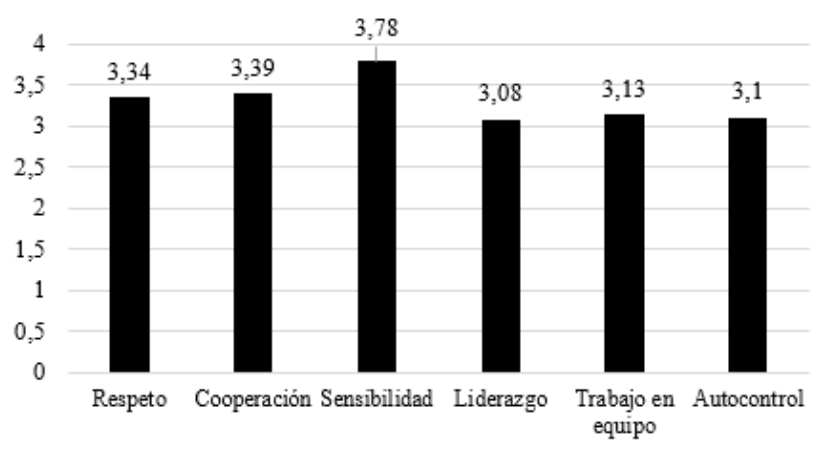

Figura 1. Carácter general del grupo-clase.

Profundizando en el análisis, y dividiendo la muestra según el porcentaje de niños y niñas en cada variable evaluada, es posible observar en la Figura 2 que tanto los nińos como las niñas, puntúan de manera elevada en la sensibilidad (46\% y $41 \%$ respectivamente), mientras que el aspecto que menor puntuación obtiene es, en el caso de los niños el liderazgo (31\%), y en el caso de las niñas el trabajo en equipo $(34 \%)$.

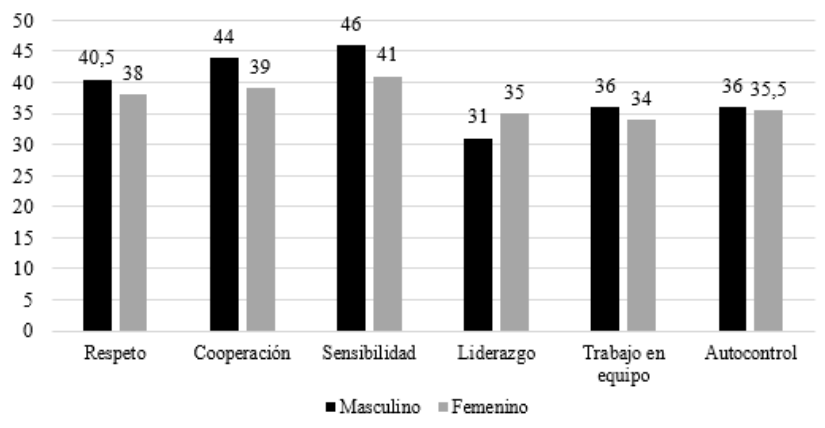

Figura 2. Carácter del grupo-clase diferenciado por sexo (\%).

En relación a los datos provenientes del TGMD, la Tabla 2 ofrece los datos derivados del análisis estadístico.

Tabla 2. Porcentaje medio del TGMD del grupo-clase.

\begin{tabular}{lccc}
\hline \multicolumn{1}{c}{ Subprueba } & Grupo-clase & Chicos & Chicas \\
\hline Locomotriz & 77.13 & 68.97 & 86.36 \\
Control de objetos & 80.83 & 84.75 & 76.54 \\
\hline
\end{tabular}

Así, en primer lugar, es posible comprobar cómo la puntuación es más elevada en la subprueba de control de objetos, frente a la subprueba locomotriz. Si dividimos el análisis por género, la tendencia se mantiene en el caso de los chicos, pero se invierte en las chicas, siendo de un $86.36 \%$ los resultados de la subprueba locomotriz y $76.54 \%$ la media de los resultados de la subprueba control de objetos.

Concretando más en el análisis de estos resultados, la Figura 3 muestra la evaluación proveniente de la subprueba locomotriz $(0-10)$ en cada una de las pruebas analizadas.

En la Figura 3 es posible observar que la puntuación más alta es obtenida por la prueba "salto con un pie" $(M=8.37)$, y la más baja "salto extendido" $(M=5.08)$. En relación al análisis por género, los chicos obtienen sus resultados más elevados en "carrera" ( $M=7.33)$ y las chicas en "salto con un pie" $(M=$ 9.82), mientras que, entre los resultados más bajos, los chicos obtienen sus peores marcas en "salto extendido" $(M=4.42)$ y las chicas en "galope" $(M=5.64)$. De manera general, la Figura 3 revela que las chicas obtienen mejores resultados que los chicos.

En relación a la subprueba control de objetos, la Figura 4 muestra los resultados obtenidos en cada una de las pruebas evaluadas, sobre 10 puntos. 


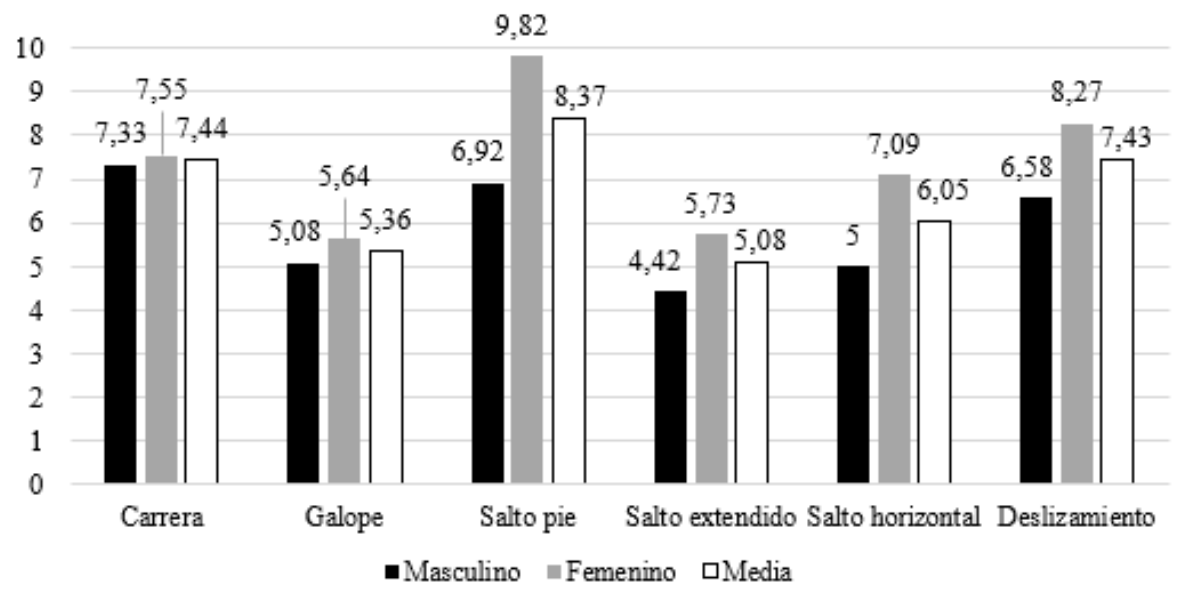

Figura 3. Subprueba locomotriz.

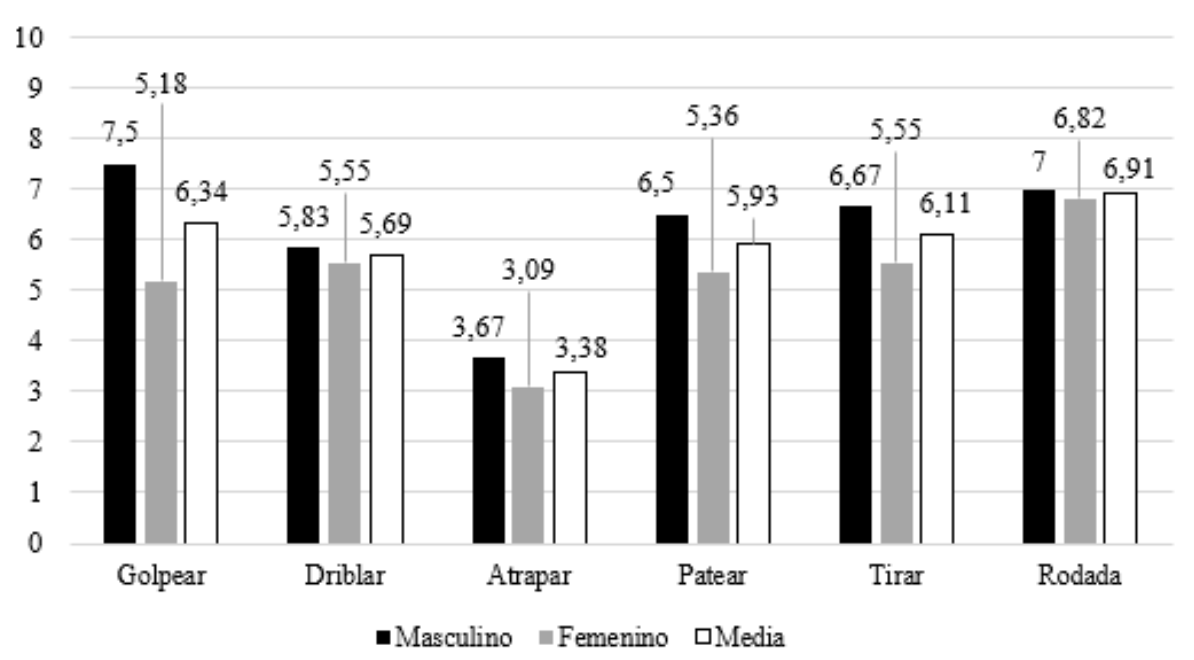

Figura 4. Subprueba control de objetos.

La Figura 4 revela que, teniendo en cuenta al grupo-clase, la media más alta es obtenida por la prueba "rodada" $(M=$ 6.91), mientras que la más baja fue marcada en la prueba de "atrapar" $(M=3.38)$. Diferenciando el análisis por género, los chicos obtuvieron su mejor puntuación en la prueba de "golpeo" $(M=7.50)$ y las chicas en "rodada" $(M=6.82)$, mientras que aquellas pruebas que obtuvieron peores resultados fueron, en ambos casos, la prueba de "atrapar", marcando un valor de 3.67 para los chicos y 3.09 para las chicas. En esta ocasión, los resultados indican mejores puntuaciones de los chicos frente a las chicas.

Finalmente, la Tabla 3 muestra el porcentaje de efectividad de la subprueba control de objetos, diferencia por género.

Tabla 3. Porcentaje de efectividad en la subprueba control de objetos.

\begin{tabular}{llllllllll}
\hline & Lanz. Bajo brazo & Lanz. Brazo & Atrapar & Driblar & Patear & Voleo bajo brazo & Voleo cabeza & Golpe corto & Golpe largo \\
\hline Masculino & 81.67 & 81.94 & 66.33 & 70.00 & 75.00 & 83.33 & 66.67 & 76.67 & 73.33 \\
Femenino & 90.91 & 75.77 & 69.09 & 72.73 & 68.18 & 76.36 & 76.36 & 72.73 & 78.18 \\
\hline
\end{tabular}

Así, es posible observar que la puntuación de los chicos más alta se encuentra en "voleo bajo brazo" (83.33\%), mientras que, en el caso de las chicas, esta es el "lanzamiento bajo brazo" (90.91\%). Por el contrario, las peores puntuaciones de efectividad fueron obtenidas en el caso de los chicos en "atrapar" (66.33\%) y en el caso de las chicas en "patear" (68.18\%). 


\section{Discusión}

El objetivo del presente trabajo ha sido conocer el estado de la motricidad gruesa y el carácter de un grupo de niños de 4 y 5 años. Para ello, se ha llevado a cabo un diseño de investigación descriptivo, con el propósito de conocer el estado de dichas variables en un momento concreto del tiempo.

Así, los resultados provenientes del análisis del carácter del grupo de niños mostraron que la sensibilidad es el aspecto que más puntuó, mientras que el liderazgo es aquello que menos. Estos resultados coinciden cuando se divide la muestra según el género, puesto que tanto los niños como las niñas, vuelven a puntuar de manera elevada en la variable sensibilidad. Sin embargo, los niños obtienen las peores puntuaciones en el liderazgo, mientras que las niñas en el trabajo en equipo, lo cual puede deberse principalmente al egocentrismo que todavía pueden poseer a estas edades (Paez \& Ramírez, 2016). Estos resultados parecen coincidir con los hallados en el trabajo de Cuesta et al. (2016), en el que los aspectos afectivo-relacionales de un grupo de niños mostraron un escaso desarrollo. Así, los resultados hallados en este trabajo parecen indicar el perfil que puede tener un niño en relación al carácter a estas edades.

Conocer estos resultados puede ser de gran utilidad para los docentes encargados de la educación de los niños a estas edades, puesto que según afirman McClenaghan y Gallahue (1985), es necesario conocer las debilidades y fortalezas de los alumnos para que así los docentes tengan posibilidades de elaborar y llevar a cabo planes de intervención ajustados a cada una de las necesidades que estos niños puedan tener, no solamente en aspectos curriculares, sino en la formación del carácter a estas edades. Por tanto, es preciso mencionar que el liderazgo y el respeto deberían ser afianzados de manera general por el grupo, y específicamente el docente debe incidir en que los niños mejoren en el liderazgo y las niñas en el trabajo en equipo y autocontrol. De igual forma que en el trabajo de Madrid et al. (2016) se consiguieron mejorar las conductas apropiadas en Educación Física y deporte, es posible la implementación de programas que consigan un desarrollo óptimo del carácter de los niños a estas edades.

En relación a los datos provenientes del TGMD, los resultados parecen indicar que los niños a estas edades, tienen mejor adquiridos el control de objetos que el aspecto locomotriz. Esta es la tendencia ofrecida en el grupo-clase de manera general. Sin embargo, en el análisis por género, los chicos obtienen mejores resultados en el control de objetos, mientras que las chicas lo hacen en el aspecto locomotriz. Esto puede ofrecer una pista para conocer de qué manera se produce el desarrollo motriz en niños y nińas a estas edades, pudiéndose comprobar que se realiza de manera distinta, lo que ajustará más específicamente aquellos programas de intervención que se pretendan realizar, como por ejemplo el llevado a cabo por
Rodríguez, Gómez, Prieto-Ayuso y Gil-Madrona (2016), cuyos efectos fueron positivos sobre un grupo de niños de $4 \mathrm{y}$ 5 ańos.

En general, estos resultados derivados de la evaluación de la motricidad gruesa, indican buenos valores de efectividad tanto en niños como en niñas, que podrían ser explicados debido a la realización de actividad física extraescolar por parte de la mayoría del grupo-clase. Estos resultados coinciden por los hallados en el trabajo de Romero, Gil-Madrona y SáezGallego (in press), cuyos resultados, tras la evaluación del desempeño psicomotriz en niños de 5 años con diferente Índice de Masa Corporal, fueron que la lateralidad marcó buenas puntuaciones. Concretando en las variables analizadas dentro de cada subprueba, dentro de la locomotriz, el salto con un pie parece configurarse como la habilidad que mayor desarrollo presenta a estas edades, mientras que en el caso de la subprueba control de objetos, la habilidad que mostró mayor puntuación en el grupo -clase fue la rodada. Estos datos nos pueden dar una idea sobre el perfil de motricidad en estas edades, pudiendo así el docente ajustar los contenidos al nivel de desarrollo del alumno. En este sentido, y debido a los resultados encontrados en este trabajo, a nivel global se debe mejorar el salto extendido y el galope, y de forma específica los nińos deberían reforzar el salto horizontal, y las nińas el salto con un pie. En cuanto a la subprueba control de objetos, tanto niños como niñas deben trabajar en el atrape.

Por tanto, se ha comprobado a lo largo de este trabajo que, para un óptimo desarrollo integral del nińo, es importante una buena evaluación motriz, como la que aquí se ha presentado, tanto a nivel afectivo-social como motriz, que permitirá al docente plantear actividades que ayuden al desarrollo de aptitudes y actitudes en el alumnado de Educación Infantil (Monge \& Meneses, 2002). Además, con este trabajo se ponen de manifiesto instrumentos de evaluación que sirven a los docentes en estas edades, con el propósito de realizar una evaluación ajustada a la edad del nińo, no solamente en un plano motor, sino también afectivo.

Por último, es preciso destacar que pese a las bondades que ofrece el trabajo, es necesario mencionar la principal limitación derivada del mismo, siendo esta la no extrapolación de los resultados a la población, debido principalmente al tamaño muestral utilizado. Así, futuros trabajos que pretendan medir la relación entre motricidad y el carácter de los niños en esta etapa educativa, deben ampliar la muestra de estudio con el propósito de salvar esta limitación, y conocer así cuál es el perfil de desarrollo emocional y motor de éstos. El presente trabajo piloto, por tanto, pretende ser un punto de partida en esta línea de investigación centrada en la evaluación de la motricidad y el carácter de los niños a estas edades. Pese a la existencia de trabajos previos relacionados con la evaluación de las habilidades sociales en estudiantes de Educación Primaria y Secundaria (Cuesta et al., 2016; Madrid 
et al., 2016; Saiz-Panadero et al., 2016), existen escasez de trabajos centrados en Educación Infantil. Por este motivo, es necesario el diseño de trabajos de investigación que aborden este objeto de estudio, como el recientemente publicado trabajo de Rodríguez et al. (2017).

\section{Conclusiones}

En virtud del objetivo de investigación marcado, se concluye que, en general, los participantes son ligeramente mejores en el control de objetos que en la subprueba locomotriz, aunque en esta última muestran más destreza las chicas, especialmente en el salto con un pie. Por su parte, los chicos son mejores en la carrera y en el control de objetos en general, destacando en el golpeo frente al género femenino. De manera general, los niños de 4 y 5 años tienen menos habilidad en el salto extendido y en la tarea de atrapar objetos. En lo que respecta al carácter, casi la mitad de los participantes puntúan alto en la sensibilidad. En relación al género, se puede apreciar que los chicos tienen menores puntuaciones en liderazgo, mientras que las chicas tienen más déficits en el trabajo en equipo.

En vistas de los resultados obtenidos, es necesario que los profesores dirijan sus esfuerzos a potenciar aquellas habilidades motrices donde los niños obtienen menores puntuaciones, así como algunos elementos referentes al carácter. De este modo podrán lograr, por un lado, un mejor desarrollo motor de los niños que favorecerá su educación integral y, por otro lado, una mejor regulación emocional que proporcione a los alumnos herramientas para enfrentar los problemas o conflictos que se dan durante el desarrollo de las actividades motrices, así como en su vida cotidiana. Para ello, es necesario que el docente diseñe actividades que estimulen el desarrollo motor y el emocional, teniendo siempre en cuenta las características individuales del sujeto.

\section{Referencias}

1. Batalla, A. (2000). Habilidades motrices. Barcelona: Inde.

2. Bornstein, M. H., Hahn, C., \& Suwalsky, J. T. D. (2013). Physically developed and exploratory infants contribute to their own long-term academic achievement. Psychological Science, 24(10), 1906-1917. doi: $10.1177 / 0956797613479974$

3. Coleto, C. (2009). Desarrollo motor en la infancia. Revista Digital de Innovación y Experiencias Educativas, 18, 1-11.

4. Cuesta, P., Prieto-Ayuso, A., \& Gil-Madrona, P. (2016). Evaluación diagnóstica en la enseńanza conjunta de habilidades sociales y motrices en Educación Infantil. Opción, 32(7), 505-525.

5. Denham, S. A., Bassett, Hideko H. H., \& Zinsser, K. (2012). Early Childhood Teachers as Socializers of Young Children's Emotional Competence. Early Childhood Education Journal, 40(3), 137-143. doi: 10.1007/s10643-012-0504-2

6. Gabbard, C., Caçola, P., Spesatto, B., \& Santos, D. (2012). The home environment and infant and young children's motor development. En A. M. Columbus (Ed.), Advances in psychology research (pp. 105-123). New York: Nova Science Publishers

7. Gil-Madrona, P. (2003). Diseño y desarrollo curricular en Educación Fisica y Educación Infantil. Sevilla: Wanceulen.

8. Gil-Madrona, P. (2004). La evaluación de la educación física en la educación infantil. Sevilla: Wanceulen.

9. Iverson, J. M. (2010). Developing language in a developing body: the relationship between motor development and language development. Journal of Child Language, 37(2), 229-261. doi: 10.1017/ S0305000909990432

10. Madrid, L., Prieto-Ayuso, A., Samalot-Rivera, A., \& Gil-Madrona, M. (2016). Extracurricular proposal evaluation for appropriate behaviors in physical education and sports. Retos. Nuevas Tendencias en Educación Física, Deporte y Recreación, 30(1), 36-42.

11. McClenaghan, B. A., \& Gallahue, D. L. (1985). Movimientos fundamentales. Su desarrollo y rehabilitación. Buenos Aires: Editorial Médica Panamericana.

12. Metcalfe, J., \& Clark, J. E. (2002). The mountain of motor development: A metaphor. En J. E. Clark \& J. H. Humphrey (Eds.), Motor development: research and reviews (pp. 163-190). Reston: NASPE Publications.

13. Monge, M. Á., \& Meneses Montero, M. (2002). Instrumentos de evaluación del desarrollo motor. Educación, 26(1), 155-168.
14. Morrison, G. S. (2005). Educación Infantil. Madrid: Pearson Espańa.

15. The New York State Association for Health, Physical Education, Recreation, and Dance (2015). Curriculum and assessment guidance document: A physical education resource. Recuperado de https://www.nysahperd. org/event.cfm?event=CurriculumDoc

16. True, L., Pfeiffer, K. A., Dowda, M., Williams, H. G., Brown, W. H., O’Neill, J., \& Pate, R. R. (2017). Motor competence and characteristics within the preschool environment. Journal of Science and Medicine in Sport, 20, 751-755. doi: 10.1016/j.jsams.2016.11.019

17. Ley Orgánica 8 de 2013. Para la mejora de la calidad educativa. Diciembre 9 de 2013. No 295.

18. Orden de 12/05/2009 [Consejería de Educación y Ciencia]. Por la que se regula la evaluación del alumnado del segundo ciclo de Educación infantil en la Comunidad Autónoma de Castilla-La Mancha. Mayo 21 de 2009.

19. Paez, A. E., \& Ramírez, D. D. R. (2016). El egocentrismo en el proceso de adaptación escolar en los niños y niñas de 3 a 4 años. Recuperado de http:// repositorio.utmachala.edu.ec/handle/48000/8379

20. Rodríguez, M. T., Gómez, I. M., Prieto-Ayuso, A., \& Gil-Madrona, P. (2017). La educación psicomotriz en su contribución al desarrollo del lenguaje en nińos que presentan necesidades específicas de apoyo educativo. Revista de investigación en Logopedia, 7(1), 89-106.

21. Romero, S. J., Gil-Madrona, P., \& Sáez-Gallego, N. M. (In press). El desempeño psicomotriz de niños de 5 años en función del índice de masa corporal.

22. Sanz-Arazuri, E., Alonso-Ruíz, R. A., Valdemoros, M. A., \& Ponce de León, A. (2013). Validación de un cuestionario que analiza cómo trabaja el profesorado de la etapa infantil la educación para la salud desde el ámbito motor. Revista Iberoamericana de Diagnóstico y Evaluación, 1(35), 9-34.

23. Saiz-Panadero, M., Prieto-Ayuso, A., Gutierrez, E. C., \& Gil-Madrona, P. (2016). Initial evaluation in 6th grade elementary teaching social skills and intervention approach school physical education. Sport TKRevista Euroamericana de Ciencias del Deporte, 5(1), 65-74.

24. Ulrich, D. (2000). Test of gross motor development-Second Edition. Examiner's manual. Austin: Pro-Ed. 